SHS Web of Conferences 7, 02005 (2014)

DOI: $10.1051 /$ shsconf / 20140702005

(C) Owned by the authors, published by EDP Sciences, 2014

\title{
Research on the Translation Language Features of Application Technology
}

\author{
Liu Xiaoshu ${ }^{1}$, Yang Lili \\ ${ }^{1}$ College of Foreign Language, Hunan Agricultural University, 410128 Changsha Hunan, China \\ ${ }^{2}$ International College, Hunan Agricultural University, 410128 Changsha Hunan, China
}

\begin{abstract}
The writing and translation of application technology literatures have played a very important role in the international process for enterprises. The scientific technologies of our country are developing rapidly, which cannot be realized without international exchange of advanced technologies. This article takes the features of operating instructions for industrial products and specialized literatures of architecture in application technology literatures as the research subject, and respectively summarizes five stylistic features of product instructions and four stylistic features of specialized literatures of architecture, expecting to provide a basis for translation work of these two kinds of application technology literatures. This article initially summarizes the writing features of operating instructions for industrial products and specialized literatures of architecture, analyzes the content points of these two kinds of application technology literatures, and then summarizes the translation standard of application technology features, interprets the technique flow of translation that has guiding significance for translation, and at last focuses on the research of translation language features of product instructions and specialized literatures of architecture, as well as provides what shall be paid attention to during the translation process of instructions and bilingual transformation technology of collocation in specialized literatures of architecture, expecting to improve the international technical exchange level for our country through the analytic process and conclusion of this article.
\end{abstract}

Keywords. application technology literatures; stylistic structural features; translation standard; translation language features; measuring technique for $\mathrm{T}$ value

\section{Introduction}

With the increasingly rapid development of economic globalization, the exchange of economy, culture, technology, etc. among the people around the world is accordingly increasing, and the translation technology plays a very important role in the intercultural exchange. According to literature property, the translation work can be divided into literary and non-literary translation, the latter of which accounts for most of the forensic texts in terms of the specific quantity. In addition, the application technology literatures are the main bridge for transnational scientific technical exchange. To provide a better translation platform for application technology literatures, it is required to conduct a research on the corresponding stylistic features and translation language features. This article explores the stylistic

This is an Open Access article distributed under the terms of the Creative Commons Attribution License 4.0, which permits unrestricted use, distribution, and reproduction in any medium, provided the original work is properly cited. 
features and translation language features of application technology literatures based on industrial products instructions and specialized literatures of architecture.

Many people have made efforts to conduct researches on features of translation technology and application technology literatures, which exactly facilitate the clearing process of translation language features of different technical literatures. Among those people: Feng Kejiang (2010) made an analysis on the stylistic features and translation for instructions, and provided a suggestion that the translation shall be based on functional translation, correctly comprehend the original text, attach importance to expression problems, and it shall be easily understood while taking into account the technical features of original texts, as well as focus on correction of the translation[1].Qin Pingxin (2011) conducted a case study on the collocation of architecture through Corpus Concordancing based on theories related to corpus linguistics, which revealed the rule of common collocation of specialized English of architecture[2]; Zhu Peiying (2013) conducted a research on specialized English literatures of architecture, proposed to focus on analysis of the structure of typical literatures in the mechanical industry and the points that shall be noted during translation, and finally described the proposed points using the case study;

This article conducts a research on the stylistic structures of operating instructions for industrial products and specialized literatures of architecture, wording and phrasing, as well as the vocabulary usage features based on the research by predecessors, expecting to explore the translation language features of application technology literatures and make contributions to facilitating the clearing process of the features of different kinds of application technology literatures.

\section{Features of these two application technology literatures}

With the rapid development of science and technology, the products and technologies are constantly updated. The reading abilities of application technology literatures for researchers and practitioners to some extent indicate the mastering abilities of new or general technologies, which will exactly facilitate the development of science and technology.

In consideration of the unique features possessed by various technical literatures and to absorb the contents of different application technology literatures, this article makes an analysis on features of operating instructions for industrial products and specialized literatures of architecture, expecting to provide a basis for their scientific translation work through the feature analysis of these two kinds of application technology literatures.

\subsection{Analysis on features of operating instructions of industrial products}

With the booming development of domestic economy and the global exchange of science and technology, which leads to increasingly prosperous products import and export, the sole representation of science and technology is product. To let the majority of users scientifically and reasonably analyze and apply the product, reading instructions will be the directest method.

Domestically, the products will be generally divided into two types--consumption products and industrial products. For the normalization, comprehensiveness and complexity of operating instructions of industrial products compared to consumption products, it is required to make an analysis on their stylistic structures, wording methods and sentence structures of interpretation to correctly and reasonably translate the instructions of industrial products.

There are five features possessed by operating instructions of industrial products:

$<$ Feature 1 $>$. Massive use of nouns and nominalization structures;

$<$ Feature $2>$. Frequent use of technical and formal terms;

$<$ Feature 3 $>$. Partial use of ellipsis structures and initialisms;

$<$ Feature $4>$. Wide use of imperative sentences and extended simple sentences;

$<$ Feature $5>$. Required to plan the structures according to product instructions.

The product instructions are mainly used to instruct users to use the product, and the content is mainly about introduction of safe use, operation principle, technical parameters, structure, installation, 
commissioning, operation and maintenance for the product with objective description. In the descriptive process, it is required to mention a great number of the names of each product component, which is the reason that $<$ Feature $1>$ is presented; Instructions of industrial product involves high-tech content, including some special cross-industrial and industrial terms, and the instruction author, as a scientific and technical personnel, has a careful thinking, and often uses formal words to present the rigor, which is the main reason that $<$ Feature $1>$ is presented; It is required to use the words as refined as possible, and this is also the requirement of expositive style of writing, which makes the article omit some articles and verbs, and non-words are to be used instead. The analysis above shall be the main reason that $<$ Feature $3>$ is presented; Industrial product has a feature of high technicality and a relatively complex structure and usage. Generally, after telling users how to operate, it is also required to make specific descriptions about the use environment, purpose, time, condition, scope and consequence for the product. Meanwhile, the instructions shall focus on introduction, and according to domestic and foreign requirements of related standard documents, only one function shall be provided in one sentence, for the purpose of avoiding the complexity and reading difficulty of description, which therefore is the main reason that $<$ Feature $4>$ is presented; $<$ Feature $5>$ is the feature presented in hierarchical division of stylistic structure and interpreted content.

\subsection{Analysis on features of specialized literature of architecture}

Specialized literature of architecture has a lot of differences from non-scientific style of writing. Its content, expression form and style features high-tech, strong specialty, strict logic and simplified expression. This section summarizes four features of specialized literature of architecture.

$<$ Feature $1>$ Architecture is a subject that combines traditional science with modern science, and its theoretical content contains combination of various subjects;

$<$ Feature $2>$ The largest feature of the specialized literature of architecture is the use of special and semi-special scientific words, many of which indicate different conceptions in different majors, and there will be even more than one explanation in the same major. The feature of using many lexical meanings, long sentences, passive sentences, lexical conversions and non-predicate verbs, as well as of strong specialty can be presented in the use of words;

$<$ Feature 3> Sometimes, the special style of writing of architecture requires some rhetorical devices to adjust the exterior-interior relations for the article, which will appropriately reflect the thought, objective things and context. When reading English, the reader is required to jump out of the structure of original text and skillfully, reasonably and greatly alter its expression form, by which the real explanation can be finally obtained;

$<$ Feature $4>$ There are many impersonal sentences in the specialized literature of architecture, and this feature is originated from its purpose to expound scientific facts, production discovery, etc.. It mainly expounds and explains the results of scientific research, proved theories or rules, and this feature can also be obviously presented in other scientific and technical articles.

The wording of the literature of architecture is generally presented in the following three aspects-objectiveness, accuracy and refinement:

Objectiveness: The objectiveness of the literature of architecture refers to the objectiveness of the discussed content. In pursuit of objective expression, passive voice and present simple tense is frequently used, and the style of writing requires simplified structure, objective expression, accurate content, large quantity of information and emphasis on existent facts instead of one behavior.

Accuracy: Accuracy refers to accurate expression, i.e. accurate wording. To accurately describe the development process and internal structure of architecture, the used sentences are generally long, and sometimes there will be just one sentence in one paragraph, because the long sentences are very persuasive and rigorous when reflecting objective things.

Refinement: The expression form of specialized literature of architecture requires simplification and refinement, and is expected to use as less words as possible to clearly express the willingness, so a lot of abbreviations will often appear in literature. The meanings of these abbreviations are easily 
understood. According to the refinement requirement, non-finite verbs, nominalized words, phrases and their simplified forms are widely used.

\section{Research on classification of translation features of application technology articles}

\subsection{Translation standard and process of translation of application technology articles}

Narrative reasoning is the main representation of application technology articles, and they generally feature direct narration, strict structure, strong logic, massive use of formulas, data and technical terms. So the translation standard of scientific and technical literature is exactness, coherence and simplicity, among which, the exactness requirement means to exactly express the connotation of original texts in the technical content, and achieve clear conception, correct logic and accurate formulas and data without any error, for the purpose of meeting the special requirements; the coherence requirement refers to correct wording and phrasing, accurate tone and expression of the translation and appropriate representation of the tone, modality, voice, tense and emphasized points; the simplicity requirement is that the translation shall be as simple and refined as possible without any redundant words, and try to be simple, clear, refined and fluent based on exactness and coherence. The above mentioned content is the objective standard of the translation of application technology articles.

The translation process can be divided into three phases--comprehension, expression, and proofreading and correction, among which the comprehension is the premise, expression the key and proofreading and correction the deepening process. The technical summary of these three phases are as follows.

1) Comprehension of the original texts is the premise of exactly expressing the translation. In this phase, it is required to take into account the whole original text, clarify the connections between words and sentences and completely comprehend the content and logical connections of original texts through the context, selection of lexical meaning and analysis of grammar. To completely comprehend the original texts, it shall be required to first consider the context, weigh the lexical meaning, then identify the grammar to clarify the logical connections, and at last comprehend the relevant content in the original texts. Therefore, the translation of sentences in the application technology literatures shall not be based on the grammatical connections only, and it also requires judgment in the logical meaning and special content;

2) In the expression phase, it is required to seek and select appropriate language materials to restate the comprehended content of the original texts. The translation quality in this phase depends on the comprehension level of the original work and the mastering degree of the language. There will be both literal and free translation. The so-called literal translation refers to using the expression technique of the original text, not only compliant with the content of the original text but also considering its form. The so-called free translation refers to using a innovative expression technique to represent the logical and image form of the original text. Free translation method will be generally used when literal translation method cannot make the article coherent;

3) Proofreading and correction phase is a deepening process of comprehension and expression. The purpose of this phase is to essentially make the translation compliant with the standard, and is also a process to further verify the content of the original text. For the application technology article requires a high accuracy and majority of formulas and data, even a slight negligence may cause a loss to the work. So the comprehension and expression shall not be finished just in one step, but shall be a gradually deepening process.

\subsection{Analysis on translation language features of operating instructions of industrial products}

Example 1 can represent the ratio of nouns and transformation of nouns to adjectives. 
Original text: Example 1. "Install the processor retention mechanism following the motherboard manufacture's installation instruction. Open the socket handle. Install the processor by carefully aligning the pins to the socket. Close the socket handle."

Translation: Example 1. “按照主板厂商的安装说明, 安装处理器固位装置。打开插座压 杆。安装处理器时小心地将插脚与插孔对齐。然后闭合插座压杆。”

The italic words in Example 1 are nouns. From the example, it can be known that the ratio of nouns is high. It is displayed in an investigation that nouns account for $30 \%$ in daily English and above $35 \%$ in scientific English. There are 30 words in the example, and nouns account for $47 \%$. The sentence also contains three nouns that are transformed into adjectives. The nouns may repeatedly appear in one sentence.

The examples shown in Table 1 indicate that nominalization structure is often used in the instructions of industrial products.

Table 1.

\begin{tabular}{|l|l|}
\hline The italic part represents the nominalization structure & Paraphrase of the corresponding italic part \\
\hline $\begin{array}{l}\text { Operation and maintenance of any welding } \\
\text { equipment involves potential hazards. }\end{array}$ & $\begin{array}{l}\text { If you operate and maintain arc welding } \\
\text { equipment, you will run potential hazards. }\end{array}$ \\
\hline $\begin{array}{l}\text { Individuals who are unfamiliar with welding } \\
\text { equipment and } \text { use faulty judgment } \text { or lack proper } \\
\text { training, may cause injury to themselves and others. }\end{array}$ & $\begin{array}{l}\text { Individuals who are unfamiliar with welding } \\
\text { equipment and judge poorly or lack proper } \\
\text { training, may injure themselves and others. }\end{array}$ \\
\hline $\begin{array}{l}\text { By turning this switch ON, a compensation circuit is } \\
\text { activated. }\end{array}$ & $\begin{array}{l}\text { When this switch turned on, a compensation } \\
\text { circuit is activated. }\end{array}$ \\
\hline Difficulty in establishing an are. & It is difficult to establish an are. \\
\hline
\end{tabular}

It can be known from the nominalization structures and the paraphrases in Form 1 that the italic phrase part of the second line in the example belongs to gerund + preposition + nominalization structure. In terms of the deep structure, the gerunds before "of" and the noun phrase after "of" belong to verb-object relation; Both the two phrases of the third line in the example belong to neutral verb + action noun structure, among which judgment and injury are abstracted from judge and injure, and they have clear action meanings; the example in the fourth line belongs to preposition + gerund structure which nominalizes the verb of turning switch, and it is equal to adverbial clause of time.

The derivatives of Latin origin and the occurrence frequency of other words with high formality in product instructions are much higher than those in non-scientific literary forms. Table 2 lists some frequently used non-technical terms and other infrequently used terms with the same meanings in product instructions.

Table 2.

\begin{tabular}{|c|c|c|}
\hline Frequently used terms & Infrequently used terms & Explanation \\
\hline application & use & 应用 \\
\hline construction & structure, building & 结构, 建造 \\
\hline commissioning & experiment and adjust & 调试 \\
\hline hazard & danger & 危险 \\
\hline optical & choice & 任选的 (选购的 $)$ \\
\hline prior to & before & $\cdots \cdots$ 之前 \\
\hline terminate & end & 结束, 终止 \\
\hline assume & take & 承担 \\
\hline vary (alter) & differ (change) & 与.....不同, 改变 \\
\hline dismantle (disassemble) & take apart & 拆卸 \\
\hline
\end{tabular}


According to the domestic and foreign requirements related to preparation of instruction documents, the style of texts in the imperative sentence shall be clear and direct, as shown in Table 3 where the method of using imperative sentences in translation from Chinese to English is listed.

Table 3.

\begin{tabular}{|c|l|l|l|}
\hline \multirow{2}{*}{$\begin{array}{c}\text { Tone that shall be } \\
\text { used }\end{array}$} & Chinese & $\begin{array}{c}\text { Translation that does not meet } \\
\text { the requirement }\end{array}$ & $\begin{array}{c}\text { Translation that meets the } \\
\text { requirement }\end{array}$ \\
\cline { 3 - 4 } & 关掉电源 & Turn off power. & $\begin{array}{l}\text { Be sure that the power has } \\
\text { been disconnected. }\end{array}$ \\
\hline $\begin{array}{c}\text { Using active voice } \\
\text { tone of voice }\end{array}$ & 不要去掉拉舌 & Do not remove Tabs. & $\begin{array}{l}\text { You should not remove the } \\
\text { tabs. }\end{array}$ \\
\hline Using action verbs & 维护、避免 & Maintain、 avoid. & Maintenance、 avoidance \\
\hline In a direct way & 拉动黑色控制杆 & Pull black lever toward you. & $\begin{array}{l}\text { User will pull the black lever } \\
\text { away from the machine. }\end{array}$ \\
\hline
\end{tabular}

\subsection{Analysis on translation language features of architecture}

To accurately translate the specialized literature of architecture, it shall start with collocation. The collocation is the important information used by words, which can reflect various common usages and combination methods for words. This article provides a measuring technique of $\mathrm{T}$ Value by which the mutual information value for collocates of the word "architecture" and statistic measuring data for $\mathrm{T}$ value are measured, and the results are listed in Table 4:

Table 4. List of statistic measuring results of MI value and T value for collocates related to architecture.

\begin{tabular}{|c|c|c|c|c|c|c|c|}
\hline Collocates & $\begin{array}{c}\text { Total } \\
\text { frequency }\end{array}$ & $\begin{array}{c}\text { Co- } \\
\text { occurrence } \\
\text { frequency }\end{array}$ & $\begin{array}{c}\text { MI } \\
\text { value }\end{array}$ & Collocates & $\begin{array}{c}\text { Total } \\
\text { frequency }\end{array}$ & $\begin{array}{c}\text { Co- } \\
\text { occurrence } \\
\text { frequency }\end{array}$ & $\begin{array}{c}\text { T } \\
\text { value }\end{array}$ \\
\hline deconstructive & 7 & 5 & 12.5 & art & 6764 & 40 & 6.20 \\
\hline metaphysic & 21 & 5 & 11.0 & modern & 5310 & 19 & 4.21 \\
\hline postmodernist & 15 & 3 & 10.7 & deconstructive & 19 & 14 & 3.74 \\
\hline thematic & 52 & 3 & 8.91 & baroque & 251 & 11 & 3.31 \\
\hline baroque & 251 & 11 & 8.52 & style & 6081 & 10 & 2.93 \\
\hline Saxon & 178 & 7 & 8.36 & English & 8537 & 10 & 2.84 \\
\hline nouveau & 107 & 3 & 7.87 & traditional & 4182 & 9 & 2.83 \\
\hline Vemacular & 191 & 3 & 7.04 & landscape & 993 & 8 & 2.79 \\
\hline gothic & 273 & 4 & 6.94 & Saxon & 178 & 7 & 2.64 \\
\hline Berkeley & 218 & 3 & 6.85 & decoration & 334 & 7 & 2.63 \\
\hline Anglo & 568 & 7 & 6.69 & Anglo & 568 & 7 & 2.62 \\
\hline landscapes & 269 & 3 & 6.54 & contemporary & 1702 & 7 & 2.57 \\
\hline Marcus & 552 & 6 & 6.51 & history & 8769 & 8 & 2.46 \\
\hline symbolic & 471 & 5 & 6.47 & Marcus & 552 & 6 & 2.42 \\
\hline masterpiece & 289 & 3 & 6.43 & urban & 1220 & 6 & 2.39 \\
\hline Georgian & 310 & 3 & 6.34 & Greek & 1326 & 6 & 2.38 \\
\hline landscape & 993 & 8 & 6.07 & church & 6934 & 7 & 2.33 \\
\hline medieval & 791 & 5 & 5.72 & arts & 2535 & 6 & 2.33 \\
\hline glorious & 660 & 4 & 5.66 & Scott & 2681 & 6 & 2.32 \\
\hline art & 6764 & 40 & 5.63 & beautiful & 4076 & 6 & 2.25 \\
\hline splendid & 739 & 4 & 5.50 & symbolic & 471 & 5 & 2.21 \\
\hline Victorian & 994 & 5 & 5.39 & medieval & 791 & 5 & 2.19 \\
\hline urban & 1220 & 6 & 5.36 & Victorian & 994 & 5 & 2.18 \\
\hline & & & & & & & \\
\hline
\end{tabular}




\begin{tabular}{|c|c|c|c|c|c|c|c|}
\hline Greek & 1326 & 6 & 5.24 & temple & 1223 & 5 & 2.17 \\
\hline contemporary & 1702 & 7 & 5.10 & classical & 1274 & 5 & 2.17 \\
\hline temple & 1223 & 5 & 5.09 & institute & 1866 & 5 & 2.14 \\
\hline classical & 1274 & 5 & 5.04 & ancient & 2046 & 5 & 2.13 \\
\hline
\end{tabular}

From the tested statistic data in Table 4, it can be known that the displayed results of measuring data for MI and T value are so different. The co-occurrence frequency of collocates with high MI value and node words is not necessarily high. It mainly depends on the ratio of co-occurrence frequency of collocates and node words to product of individual occurrence frequency.

In the translation process of specialized literature of architecture, it is easy to find that the translator has certain problems in the mastering and using of words. Translation is a cross-language and cross-cultural communication activity, and there will be both similarity and difference in different languages. One of the difficulties in the translation and conversion process is the conversion of collocation. In the translation of special English of architecture, due to the differences between English and Chinese, we cannot translate the English collocation according to Chinese collocation habit.

In summary, the translation of specialized literature of architecture requires accurate using of collocation, and application of the reasonable collocation method in the conversion of these two languages can be very helpful to the translation coherence and accuracy. So collocation is a translation language feature of specialized literature of architecture.

\section{Research conclusion and outlook}

1) To explore the translation language features of application technology, this article makes an analysis on the stylistic structure, wording and phrasing of operating instructions for industrial products and specialized literatures of architecture, expecting to provide a basis for translation work of these two application technology literature. This article also analyzes the features of different application technology literatures in different perspectives;

2) After analysis of operating instructions for industrial products and specialized literatures of architecture, this article summarizes the standard and process of translation to provide a guiding thought for technical and procedural translation;

3 ) It focuses on analysis of wording and translation language features of instructions for industrial products, which provides translation experience for one kind of instructions;

4) It focuses on analysis of translation language features of specialized literatures of architecture. It means to take different language conversion of collocation as a research object to provide a method of measuring $\mathrm{T}$ value for collocation, explain the results by mutual information value for collocation of architecture, compare the MI value measuring method and obtain the superiority of $\mathrm{T}$ value measuring method as well as the important translation language features of specialized literatures of architecture.

\section{References}

1. Feng Kejiang. Stylistic Features and Translation of Instructions[J]. Journal of Yichun College. 2010.32(11):161-164.

2. Qin Pingxin. Research on Collation of Specialized English of Architecture Based on Corpus[J]. Shandong Foreign Language Teaching Journal. 2011(1):28-33.

3. Zhu Peiying. Reference Standard of Reading for Translation Machine of Specialized Mechanical English Literatures [J]. Journal of Hubei Radio Television University. 2013.33(1):92-93.

4. Zhu Zhide. English Stylistic Features of Operating Instructions for Industrial Products and their Translation from Chinese to English[J]. Chinese Translation Journal. 2003.24(2):58-61.

5. Wang Wen, etc.. Common Linguistic Features in Medical English [J]. Medicine and Society. 2006.19(11):25-27. 
6. Zhang Shaoen, etc.. Discussion on Features and Translation of Specialized Agricultural English[J]. Agriculture Network Information. 2008(6):168-170.

7. Ding Zihua. Features and Measures of Specialized English Translation[J]. Journal of Mianyang Normal University. 2008.27(7):99-103.

8. Zhao Rong, etc.. Discussion on Subject Theoretical Basis of "Comparison and Translation of English and Chinese Language Cultures"[J]. ournal of Xi'an Foreign Languages University. 2005.13(1):40-44.

9. Zhou Yan. Application of Logic Method in Scientific English Translation[J]. Journal of University of Science and Technology Liaoning. 2012.35(1):73-76. 